\title{
Deletion of a branch-point consensus sequence in the LMX1B gene causes exon skipping in a family with nail patella syndrome
}

\author{
Jeanette D Hamlington, Mark V Clough, Jennifer A Dunston and Iain Mclntosh \\ M cKusick-N athans Institute of Genetic Medicine, Johns Hopkins University, Baltimore, USA
}

\begin{abstract}
Nail patella syndrome (NPS) has been shown to result from loss of function mutations within the transcription factor LMX1B. In a large NPS family a $17 \mathrm{bp}$ intronic deletion encompassing a consensus branchpoint sequence was observed to segregate with the NPS phenotype. RNA analysis demonstrated that deletion of the branchpoint sequence resulted in skipping of the downstream exon. A mechanism to explain this phenomenon is presented. European Journal of Human Genetics (2000) 8, 311-314.
\end{abstract}

Keywords: LMX1B; nail patella syndrome; branch-point; exon skipping; splice site mutation

\section{Introduction}

Nail patella syndrome (NPS) is a pleiotropic condition characterized by dysplasia of the nails, hypoplasia of the patellae, el bow dysplasia and progressive kidney disease. ${ }^{1}$ Recent evidence suggests that open angle glaucoma is also part of the syndrome $e^{2}$ and that other organ systems may also be affected (I Mclntosh, unpublished results). The syndrome is inherited in an autosomal dominant manner and has been shown to result from mutations in the LIM-homeodomain encoding $L M X 1 B$ gene. ${ }^{3-6}$ The $L M X 1 B$ transcription factor plays a role in defining the development of dorsal specific structures during limb development; ${ }^{7}$ its role in other organs is unclear. Analysis of over 60 LMX1B mutations in NPS families supports the hypothesis that the syndrome results from haploinsufficiency due to loss of function mutation. . $^{3-6}$

During the search for LMX1B mutations causing NPS, a $17 \mathrm{bp}$ deletion was identified upstream of the $3^{\prime}$ splice consensus which removed a consensus branchpoint sequence. The effect of this deletion on the splicing of LMX IB RNA was studied further.

\section{Materials and methods \\ Patient samples and DNA amplification}

Family 1 has been described previously. ${ }^{8}$ Genomic DNA was extracted from peripheral blood lymphocytes, PCR-amplified

Correspondence: lain McIntosh, PhD, Institute of Genetic Medicine, Johns Hopkins University, 600 North Wolfe Street / Blalock 1012G, Baltimore, MD 21287-4922, USA. Tel: +1410955 7948;

Fax: +1410614 2522; E-mail: imcintos@welch.jhu.edu

Received 27 August 1999; revised 14 October 1999; accepted

2 November 1999 and sequenced as described. ${ }^{5}$ Appropriate informed consent was obtained from all participants.

\section{RNA analysis}

Skin fibroblasts obtained from a member of family 1 with NPS were cultured and total RNA was extracted using a commercial reagent (Trizol; Life Technologies, Gaithersberg, MD, USA). RT-PCR was performed using a commercial kit (Advantage RT-PCR, Clontech, Palo Alto, CA, USA) with primers LMX-1F (5'-GACGGACTGCGCCAAG) and LMX-3anti (5'-GAAGCAGCCCAGGTGGTAC). PCR products were subjected to electrophoresis in $2 \%(w / v)$ agarose, $1 \times \mathrm{TBE}$, Southern blotted and hybridized with oligonucleotides specific for exons2 (LMX-2F: 5'-GTGTCAGCAAGCCCTCAC) and 3 (LMX-A: 5'-GCTCTTCGCGGCCAAGTGCAG). To confirm the exact nature of the splicing event, RT-PCR products were gel purified and sequenced.

\section{Results}

Mutation analysis of genomic DNA from affected members of Family 1 failed to detect any changes in coding sequence or canonical splice sites. However, a 17 bp deletion was identified starting $37 \mathrm{bp}$ upstream of exon 2 (Figure 1). The deletion removed a recognition sequence for the restriction enzyme Eagl; restriction digestion of PCR amplified DNA from 14 affected and 11 unaffected members of Family 1 indicated that the deletion was found only in persons with NPS (data not shown). The deletion was not detected in a further 50 unrelated individuals from an unaffected control population screened by Eagl digestion of PCR amplified DNA. Of interest, the deleted region included the sequence CGCTGAC 


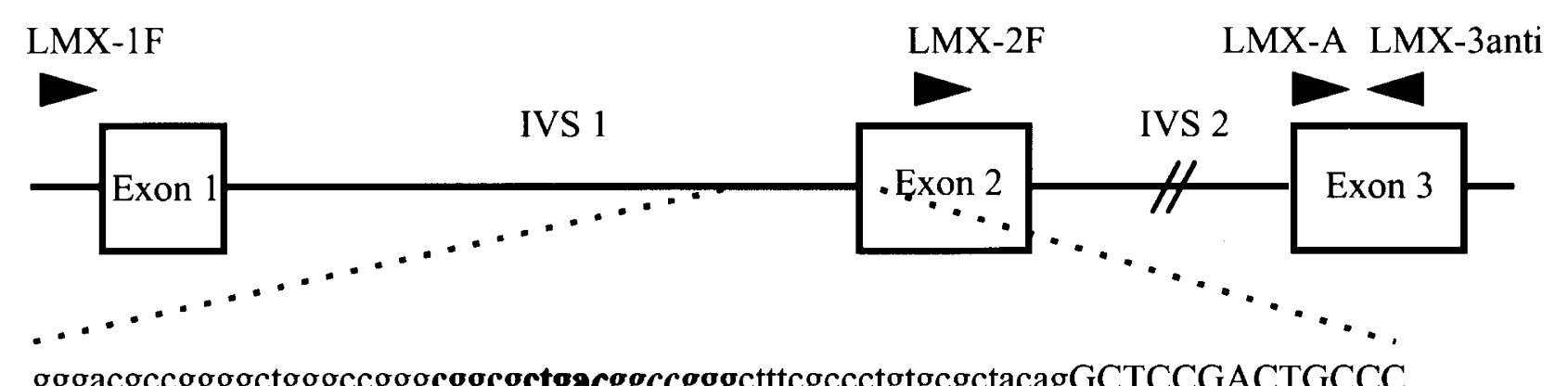

Figure 1 Schematic of the region of LM X1B discussed in the text. Exons1-3 are shown as boxes with intervening sequences as lines. The sequence around the deletion is expanded below: the deletion is in bold, the Eagl site in italics, the consensus branchpoint sequence underlined and exonic sequence in upper case. The positions and orientation of primers are shown above as arrowheads.
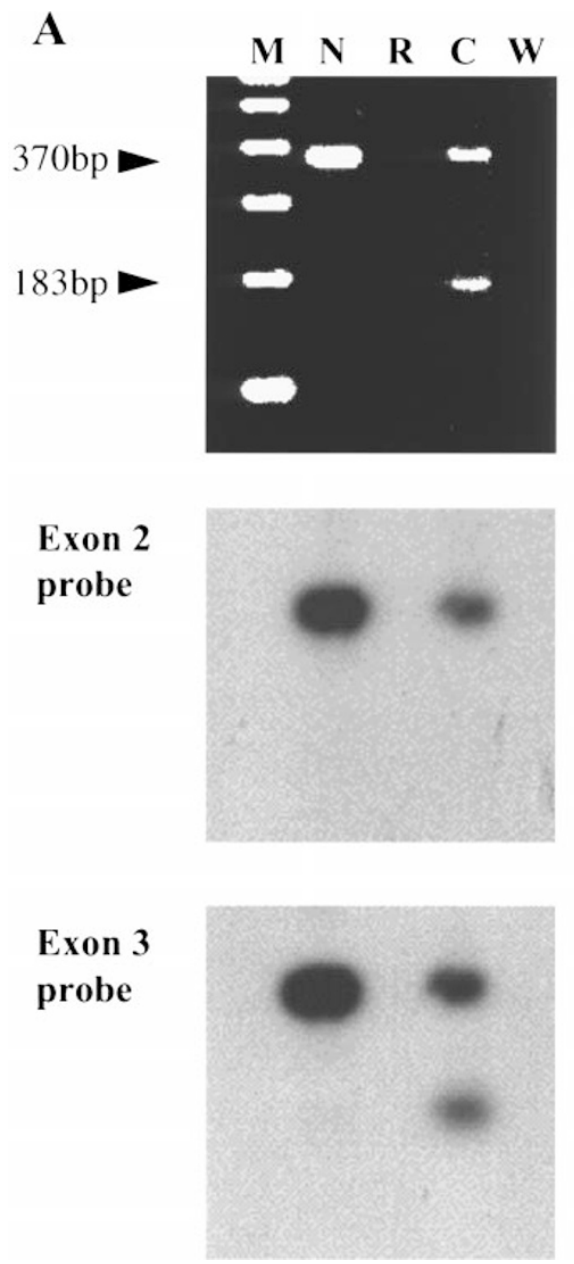

B

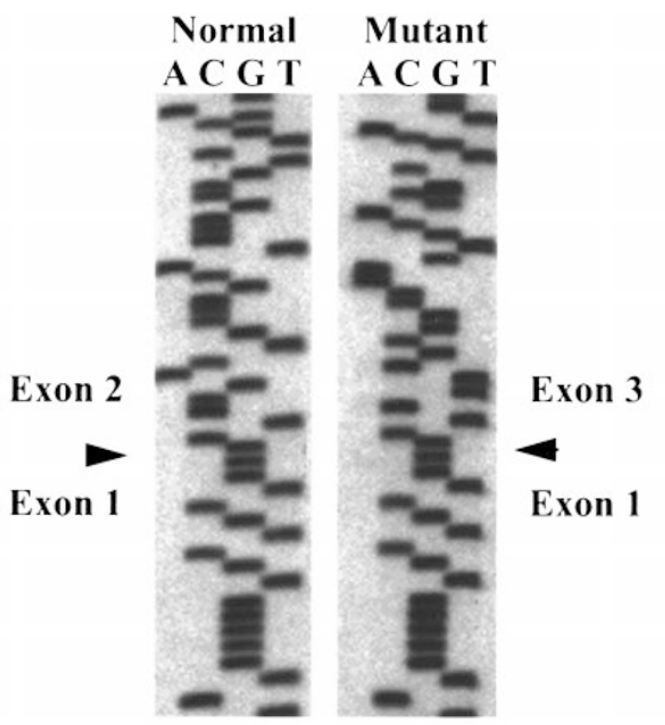

Normal $\begin{aligned} & \text { LeuGlyVal LeuLeuGlySerAspCysPro } \\ & \text { CTGGGGTGCTGCTGGGTCCGACTGCCCG }\end{aligned}$

Mutant LeuGlyValLeuLeuglySerSerArgPro CTGGGGGTGCTGCTGGGCTCTTCGCGGCCA

Figure 2 RT-PCR analysis A RT-PCR was performed as described in Materials and methods. The products of normal splicing (370bp) and with exon 2 skipped (183bp) are arrowed (upper panel). The gel was blotted and hybridized with oligonucleotides specific for exon 2 (middle panel) and exon 3 (lower panel). M: marker, 100bp ladder; N: normal control template; R: patient RNA template; C: patient cDNA template; W: no template control. B Sequence of mutant and normal PCR products. PCR products shown in A were extracted from the gel and sequenced. The exon boundary is marked with an arrowhead. The normal and mutant sequences and encoded amino acids are shown below. Exon 3 sequence is underlined. The alteration in reading frame predicts a premature termination coding $57 \mathrm{bp}$ into exon 3. 
which not only is a perfect match for the consensus for the branchpoint sequence necessary for lariat formation (YNYTRAC), but is positioned at a distance to perform such a function (within 15-40 nucleotides of the 3' splice site).

To determine the effect of this deletion on pre-mRNA splicing, total RNA was extracted from cultured skin fibroblasts, reverse transcribed and amplified using primers from exons 1 and 3. Two bands were seen upon gel electrophoresis of the PCR product from the patient's CDNA compared with a single band from control fibroblast cDNA (Figure2A). The sizes of the PCR products were those expected for a wild type transcript (upper band), and onelacking exon 2 (lower band).
This hypothesis was confirmed by Southern blotting and hybridization with probes specific for exon 2 and exon 3 (Figure2A, lower panels). To show that the splicing of exon 1 to exon 3 was precise, the PCR products were gel purified and sequenced (Figure2B). The skipping of exon 2 disrupts the reading frame leading to a premature termination codon within exon 3. Any polypeptide synthesized from this transcript would lack all functional domains and would be predicted to be non-functional. To insure that the exon skipping in this subject resulted from deletion of the branchpoint sequence, and not from any other mutation within the intron, intron 1 was amplified from an affected
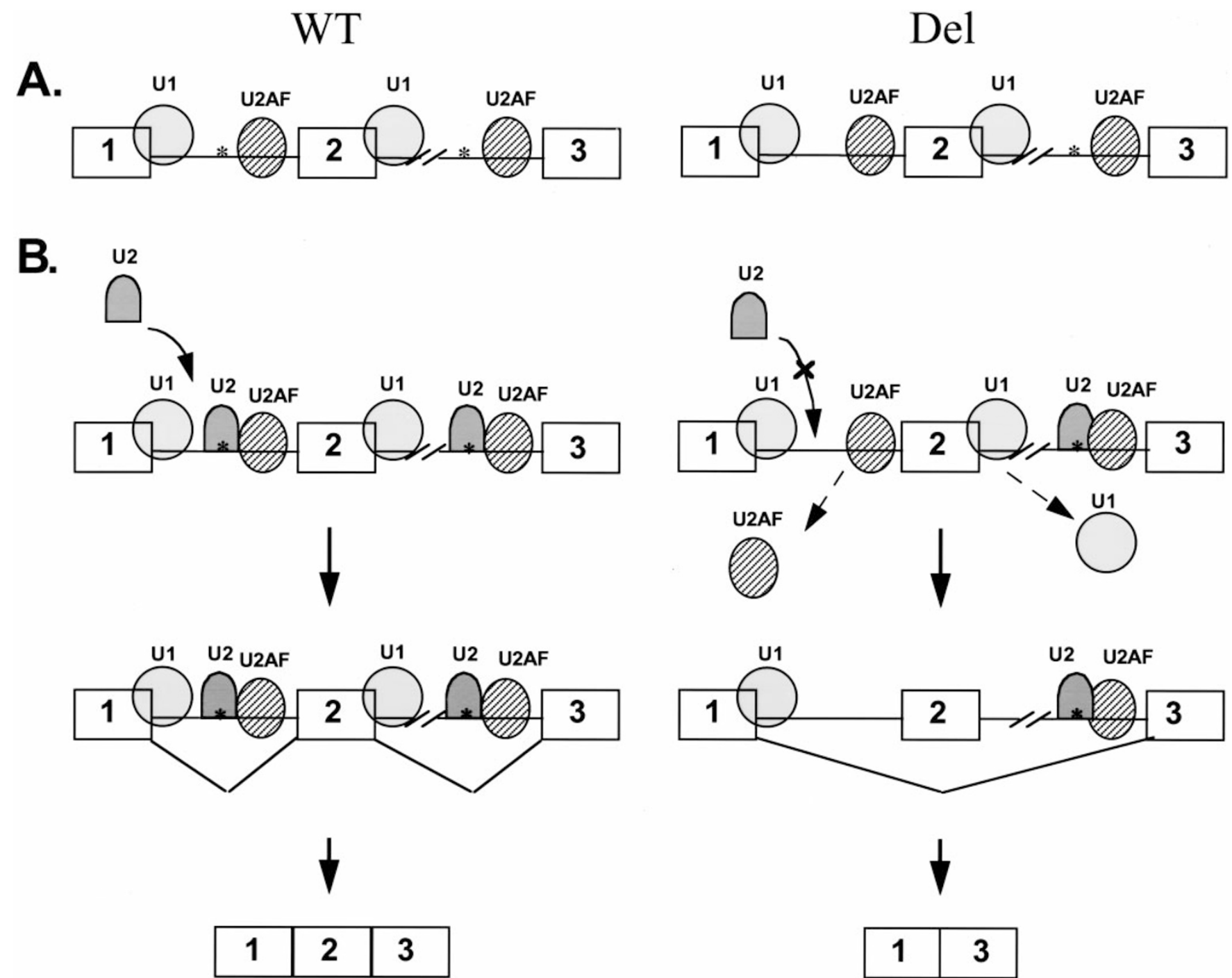

Figure 3 Model of exon skipping resulting from a branch point sequence deletion. A In both the WT and deleted variant, exon 2 boundaries are initially defined by U1 snRNP and U2AF binding to the 5' splice site consensus sequence and the polypyrimidine tract, respectively. B U2 snRNP is recruited to the spliceosome and binds the BPS. In the deleted variant, U2 snRNP is unable to bind within intron 1 due to the absence of the BPS. Consequently, destabilization of U2AF and U1 snRNP and loss of exon 2 boundary definition may occur. Remaining splicing factors promote the assembly of the active spliceosome (not shown). Splicing reactions in the WT pre-mRNA result in the joining of exons 1, 2 and 3; however, in the deleted variant, splicing reactions ligate exons 1 and 3. *branch point sequence (BPS). 
member's DNA and sequenced. No other variants were observed. The NPS phenotype in this large family is comparable to that seen in other NPS families examined. ${ }^{5,8}$

\section{Discussion}

In recent years many mutations of canonical splice sites have been reported which have been shown to affect exon definition and the splicing of premRNA, but few mutations of the branchpoint sequence have been identified. ${ }^{9}$ Such mutations have been found associated with $\mathrm{X}$-linked hydrocephalus, ${ }^{10}$ hepatic lipase deficiency, ${ }^{11}$ fish-eye disease, ${ }^{12}$ congenital contractual arachnodactyly, ${ }^{13}$ Sandh off disease, ${ }^{14}$ and Ehlers-Danlos syndrome. ${ }^{15}$ These mutations resulted in cryptic splicing, ${ }^{10,11,14}$ intron retention ${ }^{12}$ and exon skipping. ${ }^{13,15}$ That only a handful of such mutations have been identified may reflect the lack of stringency both within the branchpoint consensus, and the flexibility in distance from branch point to splice acceptor, that the splicing machinery can tolerate. In the case presented here, the GC-rich nature of the surrounding sequence may preclude the utilization of an alternate branchpoint. ${ }^{16}$

In vitro experiments have demonstrated the importance of the branchpoint sequence in the stepwise assembly of an active spliceosome complex. ${ }^{17}$ Early in spliceosome assembly, U1 snRNP and U2AF bind to the $5^{\prime}$ splice site and the polypyrimidine tract, respectively. Binding of these splicing factors and specific SR proteins defines the $5^{\prime}$ and $3^{\prime}$ boundaries of the exon and is a prerequisite for U2 SnRNP binding. ${ }^{18,19}$ Only after binding of U2 snRNP can other snRNPs such as U4, U5 and U6, assemble on the premRNA and form an active spliceosome (Figure3).

In the absence of a branchpoint sequence, stable binding of the U2 snRNP cannot occur. The initial binding of U1 snRNP and U2AF is unaffected; however, without U2 snRNP binding, the UI SnRNP and U2AF interactions with the premRNA become destabilized. ${ }^{20}$ If these interactions are destabilized across the downstream exon, the exon boundaries will not be defined. This mechanism may explain the exon skipping observed in the subject studied (Figure3). Deletion of the branchpoint sequence, in the absence of a suitable cryptic site for U2 snRNP binding, resulted in the skipping of exon 2.

\section{Acknowledgements}

The authors would like to thank the NPS family members for their participation in this study, Ms Evelyn Bull for assistance with cell culture, Dr Michael J W right for assistance in obtaining samples, and Dr Harry C Dietz for helpful discussions. This work was supported by NIH grant AR44702 and NIGMS Pre-Doctoral Training Grant GM 07814. None of the authors have any conflicts of interest.

\section{References}

1 Beals RK, Eckhardt AL: Hereditary onycho-osteodysplasia (Nailpatella syndrome). J Bone Joint Surg 1969; 51A: 505-515.
2 Lichter PR, Richards JE, Downs CA, Stringham HM, Boehnke M, Farley FA: Cosegregation of open-angle glaucoma and the nailpatella syndrome. Am J Ophthalmol 1997; 124: 506-515.

3 Dreyer SD, Zhou G, Baldini A et al: Mutations in LMX1B cause abnormal skeletal patterning and renal dysplasia in nail patella syndrome. Nat Genet 1998; 19: 47-50.

4 Vollrath D, Jaramillo-Babb VL, Clough MV et al: Loss of function mutations in the LIM homeodomain gene, LMX1B, in nail-patella syndrome. Hum Mol Genet 1998; 7: 1091-1098.

5 Mclntosh I, Dreyer SD, Clough MV et al: Mutation analysis of LMX 1B gene in nail-patella syndrome patients. Am J Hum Genet 1998; 63: 1651-1658.

6 Clough MV, Hamlington JD, Mclntosh I: Restricted distribution of loss-of-function mutations within the LMX $1 B$ genes of nail patella syndrome patients. Hum Mutat 1999; 14: 459-465.

7 Chen $\mathrm{H}$, Lun Y, Ovchimnikov D et al: Limb and kidney defects in Lmx1b mice suggest an involvement of LMX1B in human nailpatella syndrome. Nat Genet 1998; 19: 51-55.

8 Schleuterman DA, Bias WA, Murdoch JL, McKusick VA: Linkage of the loci for the nail-patella syndrome and adenylate kinase. Am J Hum Genet 1969; 21: 606-613.

9 Cooper DN, Krawczak M, Antonarakis SE: The nature and mechanisms of human gene mutation. In: Scriver CR, Beaudet AL, Sly WS, Valle D (eds). The Metabolic and Molecular Basis of Inherited Disease 7th edn. McGraw-Hill: New York, 1997, pp 259-291.

10 Rosenthal A, Jouet M, Kenwrick S: Aberrant splicing of neural cell adhesion molecule L1 mRNA in a family with X-linked hydrocephalus. Nat Genet 1992; 2: 107-112.

11 Brand K, Dugi KA, Brunzell JD, Nevin DN, Santamarina-Fojo S: A novel $A \rightarrow G$ mutation in intron 1 of the hepatic lipase gene leads to alternative splicing results in enzyme deficiency. J Lipd Res 1996; 37: 1213-1223.

12 Kuivenhoven JA, Weibusch $\mathrm{H}$, Pritchard $\mathrm{PH}$ et al: An intronic mutation in a lariat branchpoint sequence is a direct cause of an inherited human disorder (fish-eye disease). J Clin Invest 1996; 98: 358-464.

13 Putman EA, Park E-S, Aalfs CM, Hennekam RCM, Milewicz DM: Parental somatic and germ-line mosaicism for a FBN2 mutation and analysis of FBN2 transcript levels in dermal fibroblasts. Am J Hum Genet 1997; 60: 818-827.

14 Fujimaru T, Tanaka A, Choeh K, Wakamatsu N, Sakuraba H, Isshiki G: Two mutations remote from an exon/intron junction in the $\beta$-hexosaminidase $\beta$-subunit gene affect 3'-splice site selection and cause Sandhoff disease. Hum Genet 1998; 103: 462-469.

15 Burrows NP, Nicholls AC, Richards AJ et al: A point mutation in an intronic branch site results in aberrant splicing of COL5Al and in Ehlers-Danlos syndrome Typell in two British families. Am J Hum Genet 1998; 63: 390-398.

16 Ruskin B, Greene JM, Green MR: Cryptic branch point activation allows accurate in vitro splicing of human beta-globin intron mutants. Cell 1985; 41: 833-844.

17 Wassarman DA, Steitz JA: Interactions of small nuclear RNA's with precursor messenger RNA during in vitro splicing. Science 1992; 257: 1918-1925.

18 Ruskin B, Zamore PD, Green MR: A factor, U2AF, is required for U2 snRNP binding and splicing complex assembly. Cell 1988; 52: 207-219.

19 Berget SM: Exon recognition in vertebrate splicing. J Biol Chem 1995; 270: 2411-2414.

20 Champion-Arnaud P, Gozani O, Palandjian L, Reed R: Accumulation of a novel spliceosomal complex on pre-mRNAs containing branch site mutations. Mol Cell Biol 1995; 15: 5750-5756. 